\title{
Posicionamento ótimo de antenas usando Ray-Tracing e PSO
}

\author{
Emanuela Cabral, Stevan Grubisic e Walter P. Carpes Jr.
}

\begin{abstract}
Resumo-Este trabalho apresenta um modelo que associa um código de otimização baseado no método Particle Swarm Optimization (PSO) com um algoritmo que implementa a Técnica de Traçado de Raios 2D, para otimizar o posicionamento de antenas de estações radiobase em ambientes interiores. $O$ código PSO otimiza a posição da antena de modo a obter o campo mais uniforme possível em uma dada região de interesse. $O$ desempenho do código PSO foi comparado com o de um código baseado em Algoritmos Genéticos (AG), aplicado ao mesmo problema. Observou-se que o PSO apresentou resultados mais satisfatórios que o AG.
\end{abstract}

Palavras-Chave-Antenas, Particule Swarm Optimization, Traçado de Raios

Abstract-This work presents a model applied to find the best antenna base station (BS) positioning of indoor wireless systems. The model uses a Particle Swarm Optimization (PSO) code, associated to a 2D ray-tracing (RT) algorithm. The PSO optimizes the antenna location by maximizing fitness which is obtained by calling a RT algorithm that returns the lower electric field value in the regions of interest. The use of PSO in this application was compared with a validated Genetic Algorithm (GA) applied in the same problem. From simulations, we observed that PSO presented more satisfactory results than GA for this electromagnetic problem.

Keywords-Antenna, Particule Swarm Optimization, RayTracing

\section{INTRODUÇÃO}

A difusão dos sistemas de comunicação sem fio (wireless) resultou numa crescente demanda por projetos confiáveis no planejamento desses sistemas.

Nesses projetos, o posicionamento das estações radiobase (ERB) é um dos principais aspectos a se considerar para tornar a cobertura do sistema mais adequada. Quase todos os parâmetros de qualidade de um sistema wireless estão relacionados ao nível de sinal emitido pela ERB. A localização ideal é aquela que permite boa comunicação entre a ERB e todos os usuários de uma célula, sem que o nível de potência do sinal emitido pela ERB cause interferência em células próximas que compartilhem as mesmas freqüências.

Este trabalho analisa a questão de manter o melhor nível de sinal possível para todos os usuários de uma célula coberta por uma ERB. Para que esse objetivo seja alcançado, propõese maximizar o menor campo elétrico nas regiões de interesse.

Sugere-se, então, um modelo que utiliza um código de otimização baseado no método Particle Swarm Optimization

Emanuela Cabral, Stevan Grubisic e Walter P. Carpes Jr., GRUCAD, Departamento de Engenharia Elétrica, Universidade Federal de Santa Catarina, Florianópolis, Brasil, E-mails: emanuela@grucad.ufsc.br, stevan@grucad.ufsc.br, carpes@grucad.ufsc.br. Este trabalho foi parcialmente financiado pelo $\mathrm{PIBIC/CNPq}$.
(PSO) associado à Técnica de Traçado de Raios 2D (RT) para determinar o campo elétrico em todas as regiões de interesse de uma célula utilizando Ótica Geométrica (OG) e a Teoria Uniforme da Difração (TUD).

Com o intuito de validar o otimizador PSO proposto neste tipo de aplicação, analisou-se um cenário interior (indoor), onde a posição ótima de uma ERB deveria ser encontrada para prover um nível de sinal apropriado a todos os usuários de uma rede. Foram simulados tanto o modelo presente PSO/RT quanto o modelo proposto em [1], o qual utiliza Algoritmos Genéticos (AG) associado à técnica RT. Como será mostrado adiante, o modelo proposto PSO/RT forneceu melhores resultados.

\section{Modelo Proposto}

Embora as técnicas utilizadas para desenvolver o modelo sejam conhecidas, a sua associação para resolução de problemas de posicionamento de antenas em ambientes indoor é uma aplicação inédita.

O PSO é um método de otimização estocástico desenvolvido por Kennedy e Eberhart [2]. Como tem se destacado na literatura por ser um método eficiente para problemas em Eletromagnetismo, ele foi escolhido para ser utilizado no modelo proposto neste trabalho.

Similar ao AG, o PSO é inicializado com uma população de soluções aleatórias (partículas). A cada partícula potencial $\left(x_{i d}\right)$ é associada uma velocidade aleatória $\left(v_{i d}\right)$. Após a primeira avaliação do mérito (fitness) associado a cada partícula, são armazenadas as coordenadas $\left(p_{i d}\right)$ do espaço de busca do problema, associado à melhor solução (pbest). A melhor entre as melhores soluções (gbest) e as suas coordenadas $\left(p_{g d}\right)$ são também armazenadas durante o processo. Esses dados armazenados são iterativamente atualizados.

Assim, as velocidades das partículas são alteradas de modo a explorar com inteligência o espaço de busca em cada iteração.

$$
\begin{gathered}
v_{i d}=w v_{i d}+c_{1} \operatorname{rand}()\left(p_{i d}-x_{i d}\right)+c_{2} \operatorname{rand}()\left(p_{g d}-x_{i d}\right) \\
x_{i d}=x_{i d}+v_{i d}
\end{gathered}
$$

O PSO utilizado neste modelo adota as constantes de aceleração $\left(c_{1}\right.$ e $\left.c_{2}\right)$ ambas iguais a 2 [3] e um peso de inércia (w) nulo.

Para o problema apresentado, correspondente ao posicionamento de uma ERB, é necessário avaliar os campos em regiões de interesse, onde os usuários podem ser conectados a uma rede sem fio. O fitness para cada posição de uma ERB corresponde ao menor valor do campo 
elétrico nessas regiões e é fornecido pelo algoritmo RT. Maximizando-se o menor campo nessas regiões prover-se-á a todos os usuários um bom nível de sinal.

A Técnica de Traçado de Raios é um método assintótico muito utilizado na predição de problemas de cobertura, apresentando ótimos resultados em sistemas de altas frequiências. Sua formulação considera os caminhos mais relevantes que deixam a ERB e alcançam pontos de recepção. Após a definição dos caminhos, realiza-se o somatório dos campos correspondentes que são computados utilizando-se OG/TUD. O algoritmo RT usado baseia-se na Teoria de Imagens, que permite computar apenas os campos nas regiões de interesse.

O modelo proposto visa a aplicação em ambientes indoor, onde os principais mecanismos envolvidos são reflexão e transmissão através de obstáculos. Nesses ambientes, é possível desconsiderar os efeitos de difração, o que permite reduzir o tempo computacional que é proporcional à quantidade de vezes que o algoritmo RT é chamado pelo programa de otimização PSO. Assim, apenas a OG foi adotada para o cálculo dos campos neste problema.

Para um dado ponto de recepção, o campo elétrico total é obtido pela soma dos campos devidos a todos os caminhos considerados, onde o campo devido a cada caminho é dado por:

$$
E_{R}=A_{s} E_{0} e^{-j \beta s}\left\{\prod_{i} \Gamma_{i}\right\}\left\{\prod_{k} T_{i}\right\}
$$

onde $A_{s}=\rho /(s+\rho)$ é o fator de espalhamento, $s$ é a distância total (no ar) de todo o percurso a partir de um ponto de referência até o receptor; $\rho$ é a distância entre o transmissor e o ponto de referência; $E_{0}$ é o campo nesse ponto de referência; $e^{-j \beta s}$ é o fator que considera a mudança da fase ao longo de toda a propagação no ar; $\Gamma_{i}$ é o coeficiente de reflexão devido à $i$-ésima reflexão e $T_{k}$ é o coeficiente de transmissão devido à $k$ ésima transmissão do raio (considerando a propagação através de obstáculos com perdas [1]).

\section{Resultados}

O modelo proposto foi simulado num cenário indoor modelado em 2D, localizado no laboratório GRUCAD/UFSC, visando otimizar o posicionamento de um access point (isto é, uma ERB) de $2.4 \mathrm{GHz}$ WLAN (Wireless Local Área Network IEEE $802.11 \mathrm{~g}$ ), utilizada nesse ambiente.

Os pontos de recepção selecionados não correspondem ao cenário completo, mas somente a algumas regiões de interesse. Os obstáculos considerados no ambiente são paredes, portas e janelas, com diferentes tipos de materiais com perdas.

Para validar o modelo PSO/RT, o mesmo problema foi simulado com o AG/RT [1]. Nos dois casos, foram utilizadas regiões de interesse mais restritas em comparação àquela descrita em [1], com o intuito de diminuir o tempo de simulação. Conforme mencionado anteriormente, os parâmetros do PSO/RT foram $c_{1}=c_{2}=2$ e $w=0$. Para o caso do AG/RT, a probabilidade de crossover foi de 0,95 e a probabilidade de mutação foi considerada como 0,05 .
Para comparar os modelos, foram utilizados o mesmo número de partículas (20) e o mesmo número de iterações (70) para cada código. Assim, o processo completo de otimização realizou 1400 avaliações do algoritmo RT para os dois modelos. Para o problema analisado, a maior parte do tempo computacional foi gasto calculando os valores de campo pelo algoritmo RT.

A figura 1 ilustra o fitness da melhor partícula (gbest, para o PSO) em função do número de avaliações do RT. As curvas foram obtidas através da média dos resultados de seis simulações.

Através das curvas, observa-se que o PSO/RT converge mais rapidamente que o $\mathrm{AG} / \mathrm{RT}$.

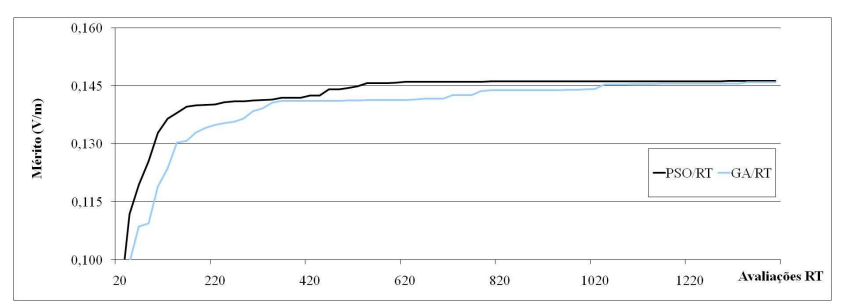

Fig. 1. Comparativo entre desempenhos PSO/RT e AG/RT

A figura 2 apresenta o mapeamento do campo elétrico no ambiente com o access point localizado na posição ótima, correspondente à figura $1: \mathrm{X}=[6,2311 ; 6,4517](\mathrm{m}) . \mathrm{O}$ tempo de cada simulação foi de aproximadamente 3 horas e 30 minutos, utilizando um computador com a seguinte configuração: 1,6 GHz, processador Intel Dual-Core, com 1 $G B$ de memória RAM e um $H D$ de $80 \mathrm{~GB}$.

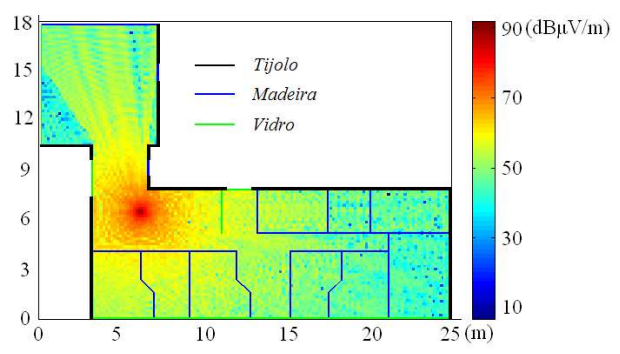

Fig. 2. Mapeamento do campo correspondente ao posicionamento ótimo da antena

\section{Conclusões}

O modelo PSO/RT apresentado mostrou-se eficiente quando utilizado em aplicações wireless em ambientes indoor. $\mathrm{O}$ PSO/RT comparado ao AG/RT apresentou melhor desempenho em termos de convergência.

\section{REFERÊNCIAS}

[1] S. Grubisic, W. P. Carpes Jr. and J. P. A. Bastos, ”Optimization Model for Antenna Positioning in Indoor Environment Using 2D Ray-Tracing Technique Associated to a Real-Coded Genetic Algorithm", IEEE Trans. on Magnetics, vol. 45(6): pp 1626-1629, Mar. 2009.

[2] J. Kennedy and R. Eberhart, "Particle swarm optimization", IEEE International Conference on Neural Networks, vol. 4, pp. 1942-1948, 27 Nov 1 Dec, 1995.

[3] R. C. Eberhart and Y. Shi, "Particle Swarm Optimization: Developments, Applications and Resources", Congress on Evolutionary Computation, 2001, vol. 1, pp. 81-86, 27-30 May, 2001. 